\title{
An effective approach for managing power consumption in cloud computing infrastructure
}

\begin{abstract}
Cloud computing offers a dynamic provisioning of server capabilities as a scalable virtualized service. Big datacenters which deliver cloud computing services consume a lot of power. This results in high operational cost and large carbon emission. One way to lower power consumption without affecting the cloud services quality is to consolidate resources for reducing power. In this paper, we introduce a DNA-based Fuzzy Genetic Algorithm (DFGA) that employs DNA-based scheduling strategies to reduce power consumption in cloud datacenters. It is a power-aware architecture for managing power consumption in the cloud computing infrastructure. We also identify the performances metrics that are needed to evaluate the proposed work performance. The experimental results show that DFGA reduced power consumption when comparing with other algorithms. Our proposed work deals with real time task which is not static, and concentrates on the dynamic users since they are involved in cloud.
\end{abstract}

Keyword: Cloud computing; DNA-based fuzzy genetic; DFGA; Energy consumption; Resource utilization; VM consolidation; VM migration 